\title{
ORIGINAL ARTICLE \\ Chromosomal rearrangements and karyotype evolution in carnivores revealed by chromosome painting
}

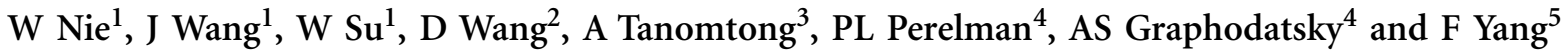 \\ Chromosomal evolution in carnivores has been revisited extensively using cross-species chromosome painting. Painting probes \\ derived from flow-sorted chromosomes of the domestic dog, which has one of the most rearranged karyotypes in mammals and \\ the highest dipoid number $(2 n=78)$ in carnivores, are a powerful tool in detecting both evolutionary intra- and inter- \\ chromosomal rearrangements. However, only a few comparative maps have been established between dog and other non-Canidae \\ species. Here, we extended cross-species painting with dog probes to seven more species representing six carnivore families: \\ Eurasian lynx (Lynx lynx), the stone marten (Martes foina), the small Indian civet (Viverricula indica), the Asian palm civet \\ (Paradoxurus hermaphrodites), Javan mongoose (Hepestes javanicas), the raccoon (Procyon lotor) and the giant panda \\ (Ailuropoda melanoleuca). The numbers and positions of intra-chromosomal rearrangements were found to differ among these \\ carnivore species. A comparative map between human and stone marten, and a map among the Yangtze finless porpoise \\ (Neophocaena phocaenoides asiaeorientalis), stone marten and human were also established to facilitate outgroup comparison \\ and to integrate comparative maps between stone marten and other carnivores with such maps between human and other \\ species. These comparative maps give further insight into genome evolution and karyotype phylogenetic relationships among \\ carnivores, and will facilitate the transfer of gene mapping data from human, domestic dog and cat to other species.
} Heredity (2012) 108, 17-27; doi:10.1038/hdy.2011.107; published online 16 November 2011

Keywords: carnivores; dog; porpoise; chromosome painting; inversion; karyotypic evolution

\section{INTRODUCTION}

Carnivores have sharply contrasting genome organizations, and are among the best examples for studying the role of chromosomal rearrangements in speciation. Previous G-banding comparisons in Carnivora have demonstrated that the karyotypes of most carnivores are highly conserved with exceptions in Ursidae and Canidae (Wurster-Hill, 1973; Wurster-Hill and Gray, 1975; Wurster-Hill and Centerwall, 1982; Dutrillaux and Couturier, 1983). Although the phylogenetic relationships in Carnivora have undergone frequent revisions (see Eizirik et al., 2010 and references therein), all available molecular evidence supports the monophyly of Carnivora, which consists of two monophyletic groups, Feliformia and Caniformia. The suborder Feliformia now includes seven families: Felidae, Hyaenidae, Viverridae, Herpestidae, Prionodontidae (Asian linsangs), Eupleridae (Malagasy carnivores) and the monotypic Nandiniidae (the African palm civet); the suborder Caniformia usually consists of Canidae, Ursidae, Procyonidae, Mustelidae, Ailuridae, Mephitidae (skunks), Otariidae, Odobenidae and Phocidae (Eizirik et al., 2010).

During the last two decades, chromosome-specific painting probes have been made for nine carnivores: the domestic cat (Felis catus, FCA) (Wienberg et al., 1997), the domestic dog (Canis familiaris, CFA) (Breen et al., 1999a; Yang et al., 1999; Graphodatsky et al., 2000a), the red fox (Vulpes vulpes, VVU) (Yang et al., 1999), the
Japanese raccoon dog (Nyctereutes procyonoides, NPR) (Nash et al., 2001), the American mink (Mustela vision, MVI) (Graphodatsky et al., 2002), the stone marten (Martes foina, MFO) (Nie et al., 2002), the giant panda (Ailuropoda melanoleuca, AME) (Nash et al., 1998), the striped skunk (Mephitis mephitis, MME) and the hooded skunk (Mephitis macroura, MMA) (Perelman et al., 2008). A series of comparative chromosome maps have been established among carnivores, and karyotypic phylogenetic relationships in different carnivore groups have been revisited by chromosome painting (Nash et al., 1998, 2001, 2008; Graphodatsky et al., 2000a, 2001, 2002, 2008; Nie et al., 2002; Tian et al., 2004; Perelman et al., 2005, 2008). Up to now, about 40 species representing most carnivore families have been studied by cross-species chromosome painting. Such studies allow a genomewide view of inter-chromosomal rearrangements and the proposition of putative ancestral karyotypes for the entire order and some families (Nash et al., 1998, 2001, 2008; Graphodatsky et al., 2001, 2002, 2008; Murphy et al., 2001a; Tian et al., 2004; Perelman et al., 2005, 2008). Nevertheless, comparative molecular cytogenetic studies in Carnivora so far have not taken account of both inter- and intra-chromosomal rearrangements in phylogenetic analyses.

Previous chromosome painting demonstrated that CFA, with the highest diploid number $(2 n=78)$ and the most rearranged karyotype in Carnivora, is an ideal reference species for high-resolution

${ }^{1}$ State Key Laboratory of Genetic Resources and Evolution, Kunming Institute of Zoology, the Chinese Academy of Sciences, Kunming, Yunnan, PR China; ${ }^{2}$ Key Laboratory of Aquatic Biodiversity and Conservation, Institute of Hydrobiology, the Chinese Academy of Sciences, Wuhan, Hubei, PR China; ${ }^{3}$ Department of Biology, Faculty of Science, Khon Kaen University, Khon Kaen, Thailand; ${ }^{4}$ Institute of molecular and cellular biology, SB RAS, Novosibirsk, Russia and ${ }^{5}$ Wellcome Trust Sanger Institute, Wellcome Trust Genome Campus, Hinxton, Cambridge, UK

Correspondence: Dr W Nie, State Key Laboratory of Genetic Resources and Evolution, Kunming Institute of Zoology, The Chinese Academy of Sciences, Kunming 650223, Yunnan, PR China.

E-mail: whnie@mail.kiz.ac.cn or Dr F Yang, Wellcome Trust Sanger Institute, Wellcome Trust Genome Campus, Hinxton, Cambridge CB10 1SA, UK.

E-mail: fy1@sanger.ac.uk

Received 30 August 2011; revised 22 September 2011; accepted 26 September 2011; published online 16 November 2011 

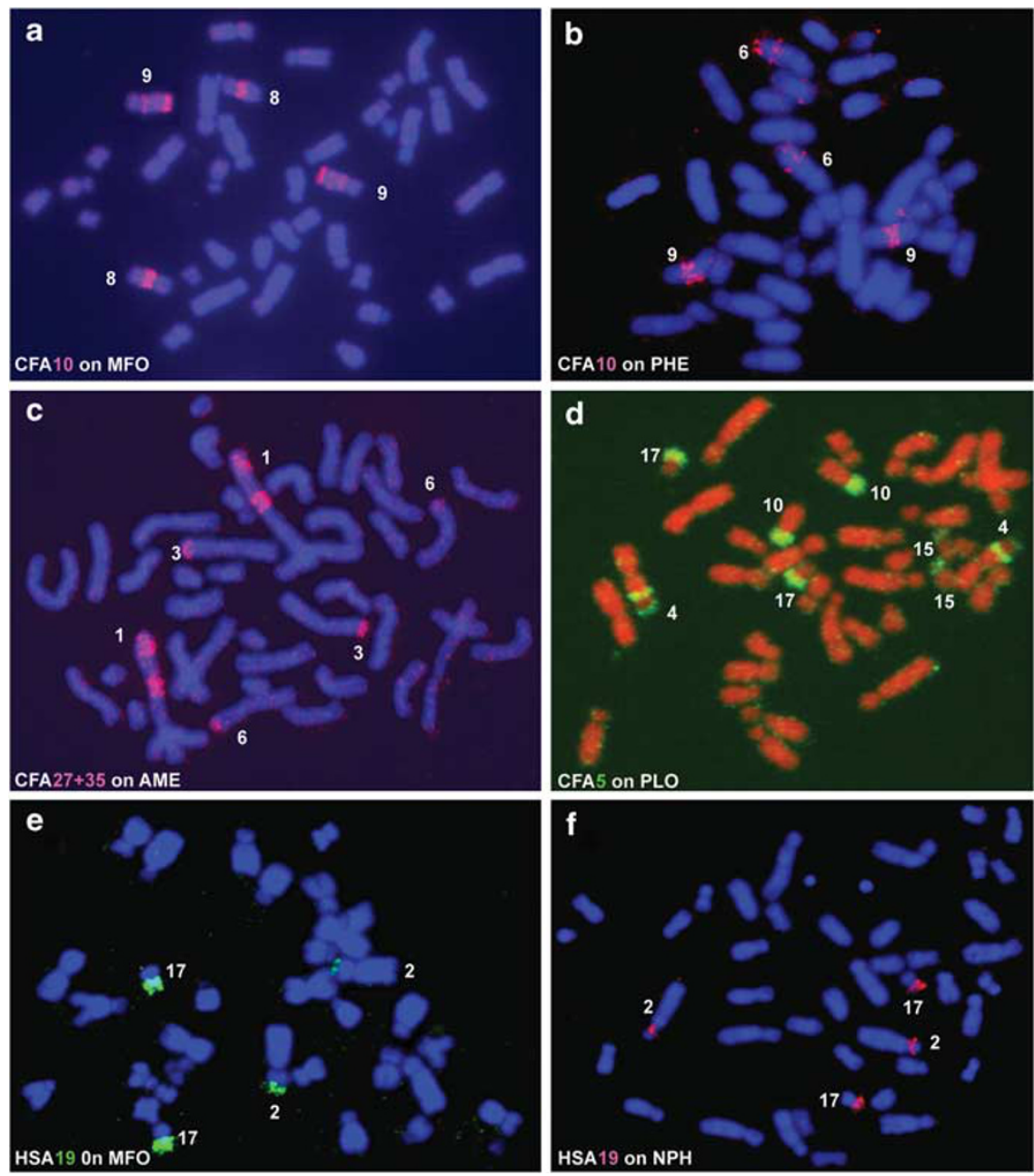

Figure 1 Examples of cross-species chromosome painting with human (HSA) and dog (CFA) chromosome-specific painting probes. (a) Hybridization of HSA 19 probe to metaphases of stone marten (MFO). (b) Hybridization of HSA 19 probe to metaphases of Yangtze finless porpoise (NPH). (c) Hybridization of CFA 10 probe to metaphases of stone marten (MFO). (d) Hybridization of CFA 10 probe to metaphases of Asian palm civet (PHE). (e) Hybridization of CFA $27+35$ probes to metaphases of giant panda (AME). (f) Hybridization of CFA 5 probe to metaphases of raccoon (PLO).

comparative genomic analysis of carnivores (Yang et al., 1999, 2000), and that CFA painting probes could reveal cryptic inversions that would have escaped detection using painting probes from species with synteny-conserved genomes such as human (Homo sapiens, HSA) and FCA (Yang et al., 2000). CFA painting probes have been widely used to establish comparative chromosomal maps between CFA and other canids (Yang et al., 1999; Graphodatsky et al., 2000a, 2001, 2008; Nie et al., 2003). Nevertheless, only eight species from other families were studied using CFA painting probes, including FCA $(2 n=38$, Yang et al., 2000), African lion (Panthera leo, PLE, $2 n=38$ ) and the clouded leopard (Neofelis nebulosa, NNE, $2 n=38$ ) (Tian et al., 2004) in Felidae; the spotted hyena (Crocuta crocuta, CCR, $2 n=40$ ) in Hyaenidae (Perelman et al., 2005); the masked palm civet (Paguma larvata, PLA, $2 n=44)$ in Viverridae (Perelman et al., 2005); Malayan sun bear (Helartos malayanus, HMA, $2 n=74$ ) (Tian et al., 2004) and the spectacled bear (Tremarctos ornatus, TOR, $2 n=50$ ) (Yang and Graphodatsky, 2004) in Ursidae; red panda (Ailurus fulgens, AFU, $2 n=36)$ in Ailuridae (Tian et al., 2002) and MVI $(2 n=30)$ in Mustelidae (Graphodatsky et al., 2000b). In addition, Nash et al. (2008) also established comparative chromosome maps between NPR, another species from the Canidae, and three carnivores (ringtails,
Bassariscus astutus, BAS, $2 n=38$; Dwarf mongoose, Helogale parvula, HPA, 2n=36; Malagasy civet, Fossa fossa, FFO, $2 n=42$ ). More carnivores representing different families remain to be studied by chromosome painting using CFA probes or probes from other carnivore species with highly rearranged karyotypes.

Molecular phylogenetic studies placed Carnivora, together with Eulipotyphla, Pholidota, Chiroptera, Perissodactyla and Cetartiodactyla, in one superordinal clade called Laurasiatheria (Murphy et al., 2001b). Within the Laurasiatheria, Pholidota, Perissodactyla and Cetartiodactyla were considered to be sister clades of Carnivora, and Pholidota was the closest living relatives of carnivores (Murphy et al., 2001b). In some molecular phylogenetic studies of Carnivora, the pangolins, whales and moles were selected as outgroups (Yu et al., 2004; Eizirik et al., 2010). Comparative chromosome maps between HSA and 10 carnivores, including FCA (Rettenberger et al., 1995; Wienberg et al., 1997; Yang et al., 2000), CFA (Breen et al., 1999b; Yang et al., 1999; Sargan et al., 2000), MVI (Hameister et al., 1997), the harbor seal (Phoca vitulina, PVI, Frönicke et al., 1997), AME (Nash et al., 1998), the domestic ferret (Mustela putorus furo, MPU, Cavagna et al., 2000), CCR (Perelman et al., 2005), PLA (Perelman et al., 2005), MME (Perelman et al., 2008) and the northern raccoon (Procyon lotor, 


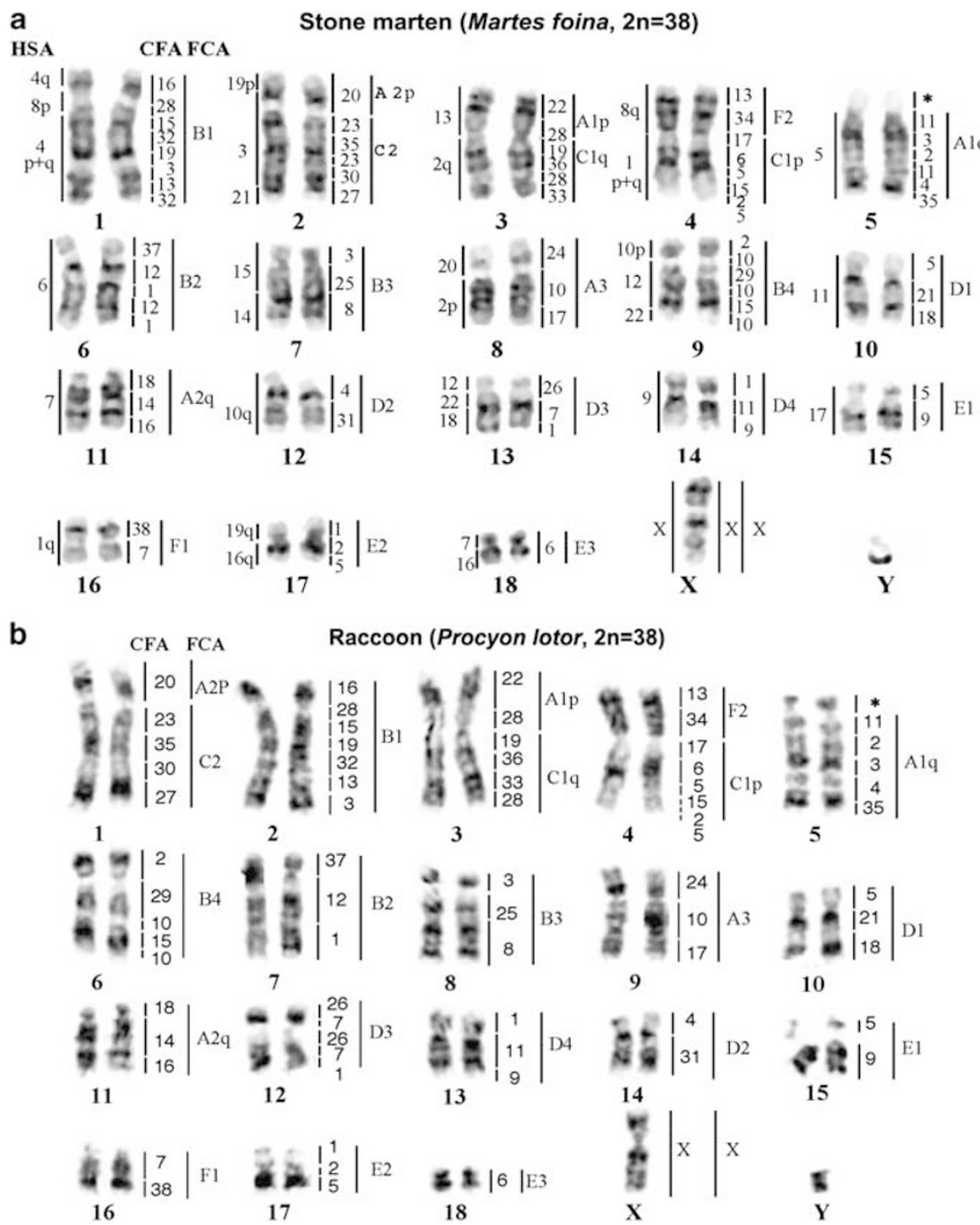

Figure 2 (a) G-banded karyotype of stone marten (MFO) with the assignment of homologies to human (HSA), dog (CFA) and cat (FCA) chromosomes. ${ }^{*}$ The segment is not painted by any dog probes. (b) G-banded karyotype of raccoon (PLO) with the assignment of homologies to dog (CFA) and cat (FCA) chromosomes. *The segment is not painted by any dog probes.

PLO, Perelman et al., 2008), and between HSA and at least one representive of all other orders in Laurasiatheria (see Yang et al., 2006 and references therein) have been established; within Laurasiatheria only two non-carnivore species, that is, the domestic pig (Sus scrofa, $2 n=38$ ) and Javan pangolin (Manis javanica, $2 n=38$ ) have been studied with probes from carnivores (CFA and MFO) (Biltueva et al., 2004; Yang et al., 2006). Although the Caniformia and Feliformia could each act as an outgroup for the other branch owing to Carnivora splitting into these two branches very early in the Carnivore radiation (Nash et al., 2008), comparison of chromosomal rearrangements between carnivores and outgroup species from other Laurasiatheria orders will be helpful in determining the ancestral state of chromosome rearrangements within Carnivora.

Here, we established comparative chromosome maps between CFA and seven species representing six families of Carnivora: MFO $(2 n=38)$, PLO $(2 n=38)$, AME $(2 n=44)$, Eurasian lynx (Lynx lynx, LLY, $2 n=38$ ), Javan mongoose (Herpestes javanicus, HJA, $2 n=36$ ), the small Indian civet (Viverricula indica, VIN, $2 n=36$ ) and the Asian palm civet (Paradoxurus hermaphroditus, PHE, $2 n=42$ ). We also present here the results of chromosome painting studies between
HSA and MFO, and among HSA, MFO and the Yangtze finless porpoise (Neophocaena phocaenoides asiaeorientalis, NPH, $2 n=44$ ), a freshwater Cetacea species from Cetartiodactyla. Combined with previously published chromosomal painting data, our data provide further evidence for inter- and intra-chromosomal rearrangements in the genomes of different carnivores and insights into the phylogenetic relationships of carnivores.

\section{MATERIALS AND METHODS}

Cell culture, chromosomal preparation and G-banding

Fibroblast cell lines derived from LLY (KCB 200020), MFO (KCB 92037), PLO (KCB 200224), NPH (KCB 200820), PHE (KCB 200632), VIN (KCB 85022), HJA (KCB 83003) and AME (KCB200405) were provided by Kunming Cell Bank of the Chinese Academy of Sciences, Kunming, Yunnan, China. The cells culture, chromosomal preparation and G-banding followed the methods as described previously (Nie et al., 2002).

\section{Fluorescence in situ hybridization}

Chromosome-specific painting probes for CFA, MFO and HSA were prepared by degenerate oligonucleotide-primed PCR (Telenius et al., 1992) amplification of flow-sorted chromosomes as previously described (Yang et al., 1999; 


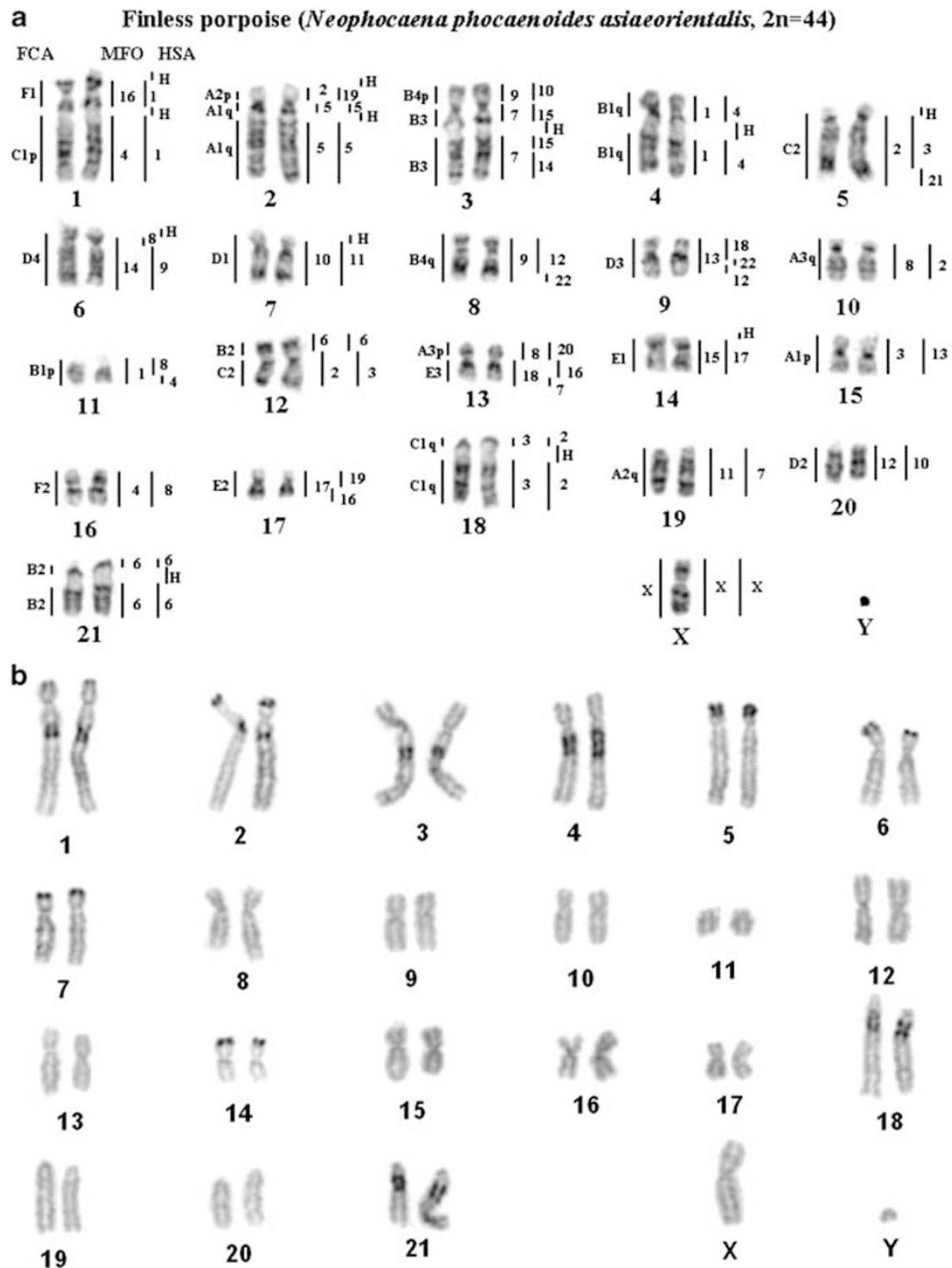

Figure 3 (a) G-banded karyotype of Yangtze finless porpoise (NPH) with the assignment of homologies to human (HSA), cat (FCA) and stone marten (MFO) chromosomes. (b) C-banded karyotype of Yangtze finless porpoise (NPH).

Nie et al., 2002). Fluorescence in situ hybridization, detection, image capture and processing were carried out following Yang et al. (2000) and Nie et al. (2002).

\section{Chromosome nomenclature}

CFA chromosome nomenclature followed Yang et al. (1999), its correspondence with Breen et al. (1999a) nomenclature has been reported by Sargan et al. (2000). Chromosomes of PHE, AME and PLO were numbered according to the G-banding karyotypes reported previously (Wurster-Hill and Gray, 1975; Nash and O'Brien, 1987; Stanyon et al., 1993). The arrangement of LLY chromosomes referred to the widely accepted FCA chromosomal nomenclature (Wurster-Hill and Centerwall, 1982). To facilitate the integration of cytogenetic maps, the chromosomes of NPH were arranged according to the nomenclature of the Atlantic bottlenose dolphin (Tursiops truncatus) (Bielec et al., 1998) and the long-finned pilot whale (Globicephala melas) (Kulemzina et al., 2009). The chromosomes of other carnivores were arranged based on their relative length, from the longest to the shortest.

\section{RESULTS}

Hybridizing HSA probes onto MFO chromosomes

Chromosomal homologies between MFO and several Mustelidae species have been established using MFO chromosome-specific probes (Nie et al., 2002). To extend the homology link with HSA to more carnivore species, the metaphase spreads of MFO were also painted with HSA chromosome paints. Hybridization example of HSA probes is shown in Figure 1a. Homologous HSA chromosomal segments are summarized to the left of each MFO chromosome (Figure 2a). The $22 \mathrm{HSA}$ autosomal probes detected 32 homologous chromosomal segments in the genome of MFO. Besides the conserved syntenic segment associations characteristic for eutherian mammals (that is, HSA $3 / 21,4 / 8,7 / 16,10 / 12 / 22,12 / 22,14 / 15$ and $16 / 19$; Murphy et al., 2001a; Yang et al., 2003), five more syntenic segment associations (HSA 1/8, 2/13, 2/20, 3/19 and 18/22/12) were found in the genome of MFO. 
Giant panda (Ailuropoda melanoleuca, $2 n=44$ )

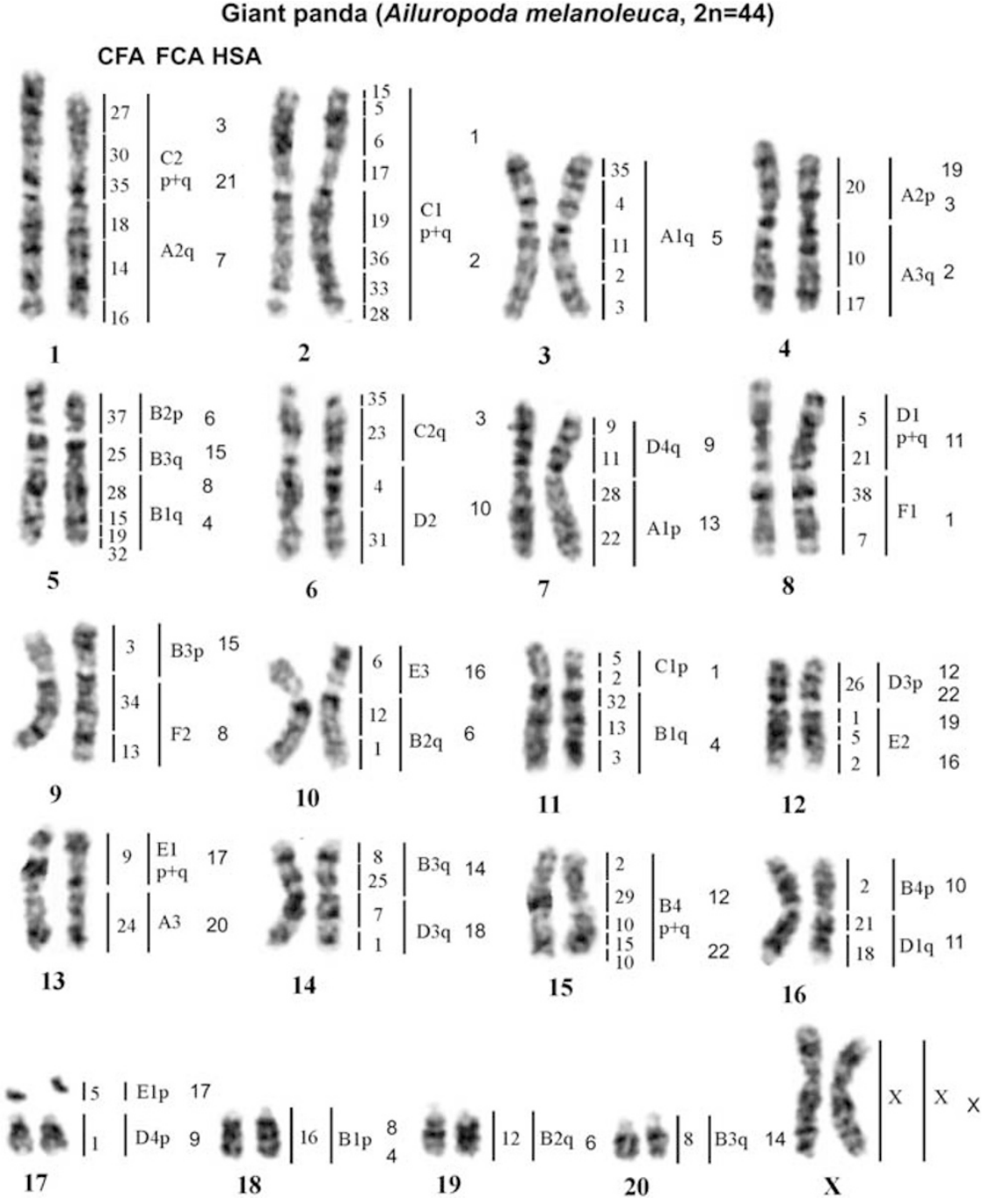

Figure 4 G-banded karyotype of giant panda (AME) with the assignment of homologies to dog (CFA), cat (FCA) and human (HSA) chromosomes. HSA painting data are cited from Nash et al. (1998).

\section{Karyotype description of the Yangtze finless porpoise and cross-species painting with HSA and MFO probes}

The Yangtze finless porpoise $(\mathrm{NPH})$ is the sole freshwater subspecies of the finless porpoise. It has a $2 n=44$ karyotype, the same as most Cetacea species (Arnason, 2006). There are 17 pairs of bi-armed and four pairs of acrocentric autosomes. The $\mathrm{X}$ chromosome is metacentric; the acrocentric $\mathrm{Y}$ is the smallest chromosome (Figure 3a). C-bands, generated by over-denaturing metaphases with $70 \%$ formamide $/ 30 \%$ $2 \times$ SSC (standard saline citrate) solution, were mainly localized at the telomeric regions of chromosomes $1,2,5-7$ and 14, while chromosomes $1-4,18$ and 21 displayed interstitial C-bands (Figure 3b).

CFA paint probes failed to work on the chromosomes of NPH after several attempts. Thus, only probes from HSA and MFO were utilized to paint its chromosomes. An example of hybridization of HSA probes is shown in Figure 1b. Hybridization patterns of HSA painting probes onto NPH chromosomes are consistent with previous data on the long-finned pilot whale ( $2 n=44$, Kulemzina et al., 2009), except that some homologous chromosome segments were disrupted by heterochromatic blocks (Figure 3a). The 22 human autosomal probes detected 40 homologous chromosomal segments in the NPH genome.
Six additional syntenic segment associations (that is, HSA $3 / 6,5 / 19$, $8 / 9,10 / 15,16 / 20$ and $18 / 22 / 12$ ) were present in the NPH genome besides those conserved syntenic segment associations ancestral for eutherian mammals. The $18 \mathrm{MFO}$ autosomal probes detected 31 homologous chromosomal segments in the NPH genome (Figure 3a). Painting probes from $10 \mathrm{MFO}$ chromosomes (\#5, 10-18) each painted one segment or one entire NPH chromosome, while the other MFO chromosome probes each gave two or three pairs of signals on NPH chromosomes. To facilitate comparison of homologous chromosomal segments among species, the homologous chromosomal segments of FCA were also indicated to the left of NPH chromosomes based on a published comparative chromosome map between MFO and FCA (Nie et al., 2002).

\section{Hybridizing CFA probes onto chromosomes of seven carnivore} species

Chromosome painting probes from CFA were hybridized to metaphase spreads of MFO, PLO, AME, LLY, HJA, VIN and PHE. Each CFA probe yielded 1 to 4 pairs of hybridization signals on the metaphase spreads of these seven species. Hybridization examples 

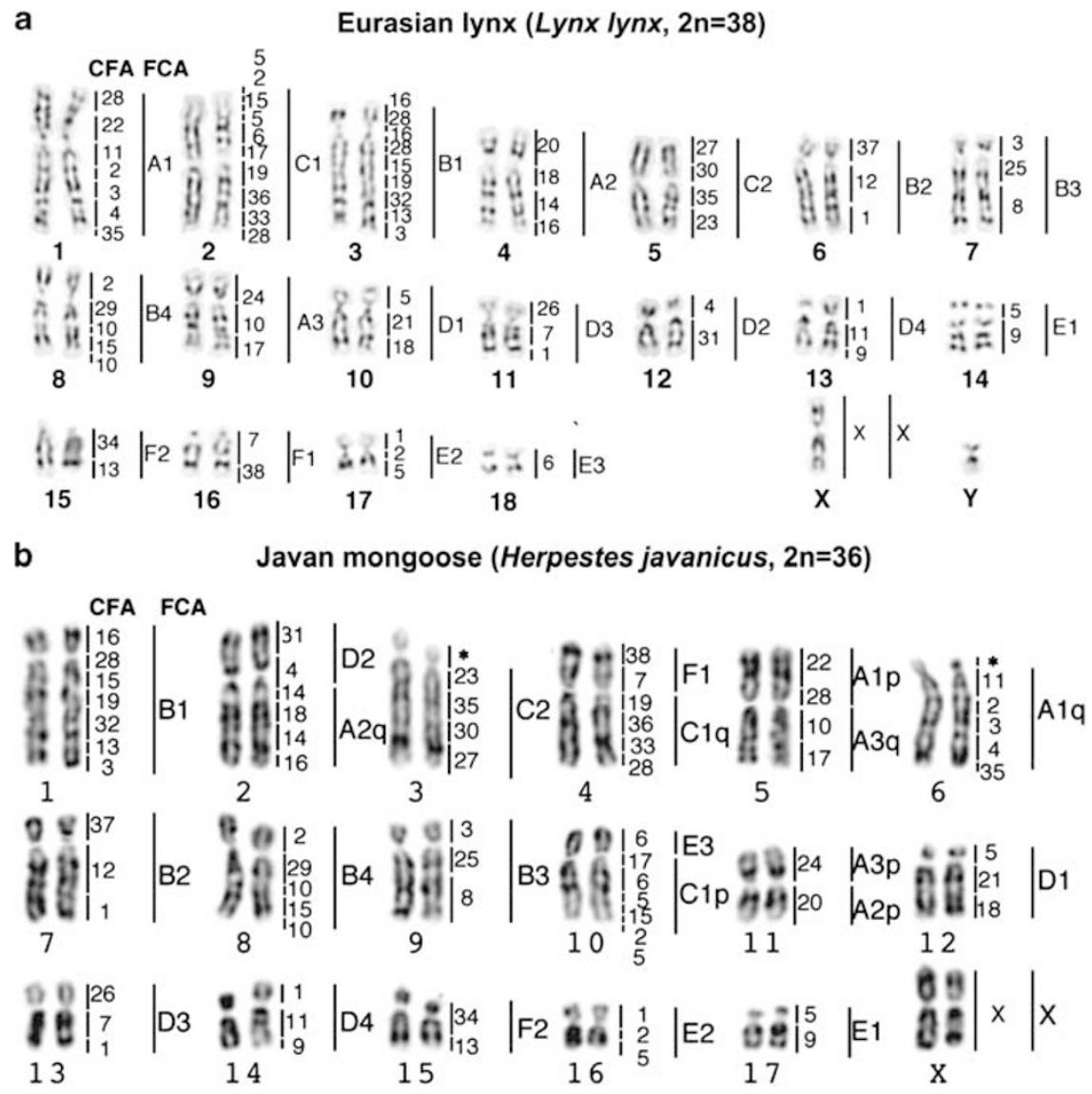

Figure 5 (a) G-banded karyotype of Eurasian lynx (Lynx lynx) with the assignment of homologies with dog (CFA) and cat (FCA) chromosomes. (b) G-banded karyotype of Javan mongoose (HJA) with the assignment of homologies with dog (CFA) and cat (FCA) chromosomes. *The segment is not painted by any dog probes.

are shown in Figures 1c-f. The hybridization patterns of $38 \mathrm{CFA}$ autosomal probes and the $\mathrm{X}$ probe were summarized onto G-banded karyotypes of these seven species. Chromosomal correspondence with FCA as inferred from the CFA-FCA comparative chromosomal map (Yang et al., 2000) was also indicated beside the CFA segments on Gbanded karyotypes. In total, 38 CFA autosomal probes revealed 72, 69, $74,69,68,68$ and 67 homologous segments in the genomes of MFO (Figure 2a), PLO (Figure 2b), AME (Figure 4), LLY (Figure 5a), HJA (Figure 5b), VIN (Figure 6a) and PHE (Figure 6b), respectively.

\section{DISCUSSION}

Implications for the signature rearrangements of Carnivora and for the putative ancestral carnivore karyotype

The establishment of comparative maps between CFA and the pig (Biltueva et al., 2004), between MFO and Javan pangolin (Yang et al., 2006), and between MFO and NPH (this study) provides a chance to compare directly chromosome homology between species in Carnivora and species in other orders from the superordinal clade Laurasiatheria, especially species used as outgroups in carnivore molecular phylogenetic studies. Of 18 MFO autosome paints, nine (MFO 10-18) and six (MFO 11, 12, 14, 15, 17 and 18) paints each hybridized to one segment or one chromosome in NPH (Figure 3a) and Javan pangolin (Yang et al., 2006), respectively. One association (MFO 6+2) seems to be common to NPH and Javan pangolin, but the results of chromosome painting with HSA probes confirmed that segments homologous to MFO 2 in this association were of different origins in NPH (homologous to HSA3) and Javan pangolin (homologous to HSA19p).

In Carnivora, together with MFO, chromosome homologies between human and 11 species have been established (Rettenberger et al., 1995; Frönicke et al., 1997; Hameister et al., 1997; Wienberg et al., 1997; Nash et al., 1998; Yang et al., 1999, 2000; Cavagna et al., 2000; Sargan et al., 2000; Perelman et al., 2005, 2008; this study). Previous comparison of maps between HSA and these carnivores suggested that the HSA 2p/20, 18/22/12 and 19/3 associations could be specific signatures for Carnivora (Murphy et al., 2001a; Perelman et al., 2008). Except for the conserved syntenic segment associations characteristic for eutherian mammals, we found no common associations between carnivores and Javan pangolin, but one common association (HSA 18/22/12) between carnivores and NPH. The HSA 18/22/12 association was also detected in the Atlantic bottlenose dolphin $(T$. truncatus, Bielec et al., 1998) and the long-finned pilot whale (G. melas, Kulemzina et al., 2009). Therefore, the HSA 18/22/12 association could not be considered as a character specific for Carnivora; instead it could be a cytogenetic character linking Carnivora and Cetartiodactyla.

Reconstruction of ancestral karyotypes of different mammalian taxa will be helpful in determining the mode and tempo of evolutionary changes that have occurred in mammalian phylogenetic lineages (Murphy et al., 2001a). Two types of ancestral carnivore karyotypes (ACKs) with different diploid numbers $(2 n=42$ and $2 n=38)$ have been proposed based on comparisons of R-banded karyotypes and fluorescence in situ hybridization data (Dutrillaux and Couturier, 


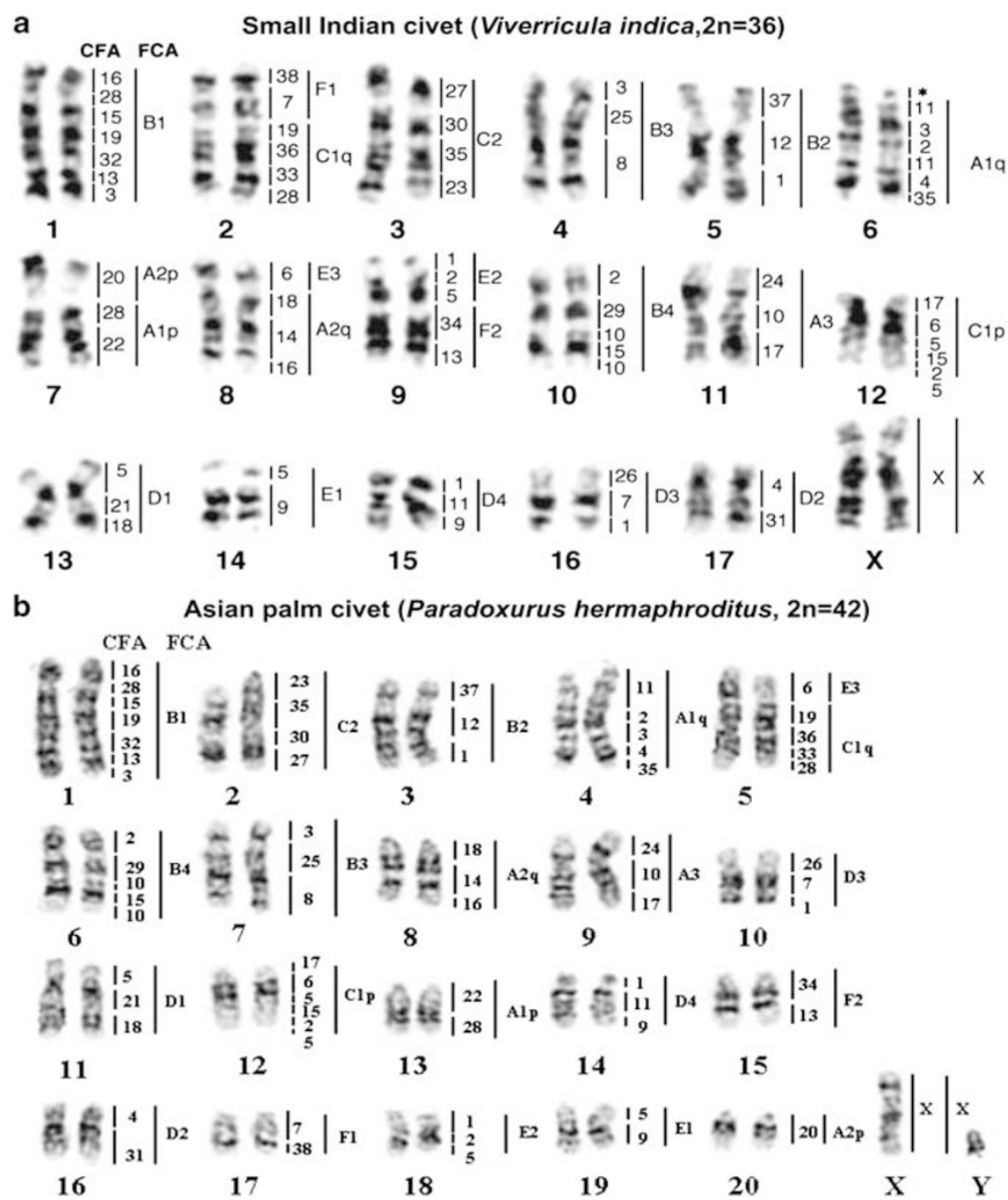

Figure 6 (a) G-banded karyotype of small Indian civet (VIN) with the assignment of homologies to dog (CFA) and cat (FCA) chromosomes. *The segment is not painted by any dog probes. (b) G-banded karyotype of Asian palm civet (PHE) with the assignment of homologies to dog (CFA) and cat (FCA) chromosomes.

1983; Frönicke et al., 1997; Murphy et al., 2001a; Nash et al., 2008). The majority of the ancestral chromosomes are identical in these two types of ACKs. The difference between $2 n=42$ and $2 n=38$ putative ancestral karyotypes concerns two chromosomes, homologous to FCA $\mathrm{A} 1 \mathrm{p}+\mathrm{C} 1 \mathrm{q}$ and $\mathrm{C} 1 \mathrm{p}+\mathrm{F} 2$. In the $2 n=42 \mathrm{ACK}$, these two chromosomes were supposed to be four single chromosomes homologous to cat A1p, C1q, C1p and F2 (Dutrillaux and Couturier, 1983; Murphy et al., 2001a), while they were retained as two whole chromosomes in the $2 n=38$ ACK (Frönicke et al., 1997; Nash et al., 2008). Recently, Perelman et al. (2008) also questioned the ancestral state of four ACK chromosomes, homologous to FCA A2p+C2, A3p+A3q, $\mathrm{A} 1 \mathrm{p}+\mathrm{C} 1 \mathrm{q}$ and $\mathrm{C} 1 \mathrm{p}+\mathrm{F} 2$, but it proved difficult to determine whether fissions or fusions of these four chromosomes represented the ancestral state when analyzing the distribution of these four chromosomes on different branches of the Carnivora tree.

Comparative maps between HSA and representatives of all the orders in Laurasiatheria have been established (see Yang et al., 2006 and references therein). Comparing these maps with those between carnivores, we found that no association was common to species in Laurasiatheria if we excluded the putative ancestral syntenic associations for eutherian mammals. Nevertheless, one fission event occurring on the chromosome homologous to FCA A3p+A3q is noteworthy. The FCA $\mathrm{A} 3 \mathrm{p}+\mathrm{A} 3 \mathrm{q}$ homologues were present as two chromosomes or chromosomal segments (that is, A3p and $\mathrm{A} 3 \mathrm{q}$ ) in species from other orders such as the pig (Cetartiodactyla, Biltueva et al., 2004), Javan pangolin (Pholidota, Yang et al., 2006) and NPH (Cetartiodactyla, Figure 3a). Furthermore, FCA A3p and A3q were also found to be homologous to two discrete chromosomal segments in HSA (that is, a representative species of Euarchontoglires, Yang et al., 1999, 2000). However, both fusion and fission states of FCA A3 were found in different carnivore families and even in species with different diploid numbers in the same family (Figure 7). These data taken together appear to support the idea that the fission state (rather than the fusion state) of FCA A3p+A3q should be regarded as the ancestral condition. In other words, chromosomal segments homologous to FCA $\mathrm{A} 3 \mathrm{p}$ and $\mathrm{A} 3 \mathrm{q}$ could represent two separate chromosomes in the ACK.

Cryptic inversions in carnivores revealed by CFA painting probes Using the CFA-FCA comparative chromosomal map (Yang et al., 2000) as the common reference and comparing the hybridization patterns of CFA painting probes on the large blocks of synteny- 


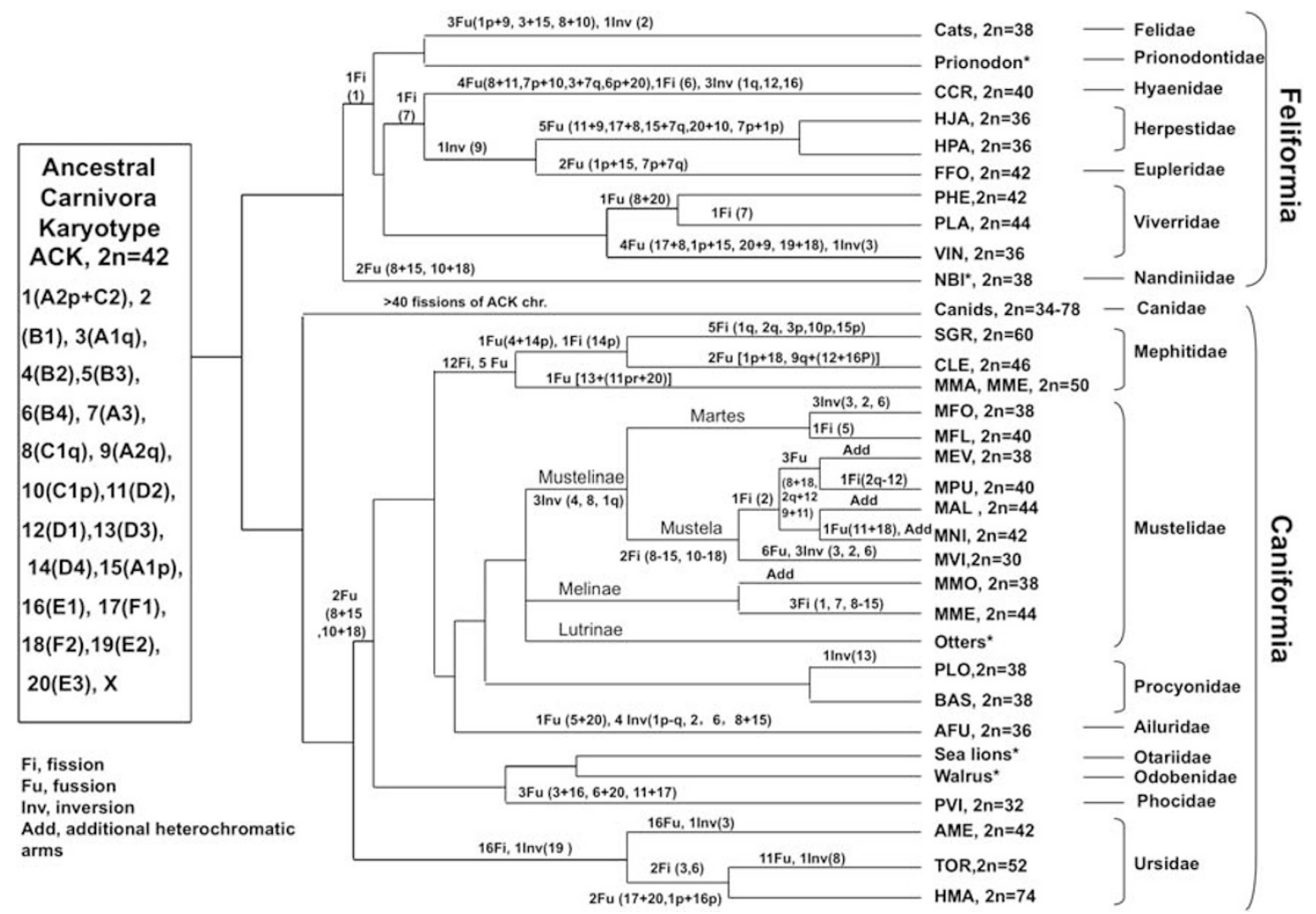

Figure 7 Karyotype relationships of the order Carnivora. Tree topology for Carnivora is modified from Eizirik et al. (2010). Published chromosome painting data for Felidae (Wienberg et al., 1997; Yang et al., 2000; Tian et al., 2004), Hyaenidae and Viverridae (Perelman et al., 2005), Herpestidae and Eupleridae (Nash et al., 2008), Canidae (Breen et al., 1999b; Yang et al., 1999, 2000; Graphodatsky et al., 2000a, 2001, 2008; Nash et al., 2001; Nie et al., 2003), Mephitidae (Perelman et al., 2008), Mustelidae (Hameister et al., 1997; Cavagna et al., 2000; Graphodatsky et al., 2000b, 2002; Nie et al., 2002), Procyonidae (Nash et al., 2008; Perelman et al., 2008), Ailuridae (Nie et al., 2002; Tian et al., 2002), Phocidae (Frönicke et al., 1997), Ursidae (Nash et al., 1998, 2001; Tian et al., 2004; Yang and Graphodatsky, 2004) and the data in this study were used for this figure. The ACK was from Murphy et al. (2001a). Chromosome numbers in brackets on the tree correspond to ACK chromosomes. ${ }^{*}$ Species in the branches have no chromosome painting data or not confirmed by chromosome painting. CLE, Conepatus leuconotus; MAL, Mustela altaica; MFL, Martes flavigula; MME, Meles meles; MMO, Melogale moschata; MNI, Mustela nivalis; MLU, Mustela lutreola; SGR, Spilogale gracilis.

conserved chromosomes (homologous FCA chromosomes or chromosomal segments), we detected cryptic inversions, with varying numbers and positions, in the genomes of MFO, PLO, AME, LLY, HJA and VIN. However, no inversions were detected in the genome of PHE. Combined analysis of the current data with the previously published data allows an overview of the inversions that occurred in carnivores belonging to different families (Table 1).

In Felidae, paints from CFA chromosomes 16 and 28 on chromosomes homologous to FCA B1 displayed the same painting pattern of CFA 16/28/16/28 in LLY (Figure 5a), FCA (Yang et al., 2000), NNE and PLE (Tian et al., 2004), but the arrangement of CFA chromosomes 16 and 28 homologous segments in other carnivores was CFA $16 / 28$, suggesting a common inversion occurred in felids.

In Herpestidae, only HJA (this study) and Dwarf mongoose (HPA) (Nash et al., 2008) have chromosome painting data. These two species have the same diploid number $(2 n=36)$ and an identical chromosomal homology pattern with FCA. An inversion was detected by CFA and NPR painting probes respectively in chromosomal segments homologous to FCA A2q in HJA (CFA 14/18/14/16, Figure 5b) and HPA (NPR 1/11/1/18, Nash et al., 2008). Chromosome painting results showed that CFA chromosomes 14, 18 and 16 corresponded to NPR chromosomes $1 \mathrm{p}, 11 \mathrm{p}$ and 18 , respectively (Graphodatsky et al., 2001). The inversion detected by the CFA and NPR painting probes in HJA and HPA appears to be the same. The same inversion was also detected in the same homology segment in Malagasy civet (FFO) by NPR painting probes (NPR 1/11/1/18, Nash et al., 2008). Previous molecular phylogenetic study indicated that Malagasy carnivores actually formed a separate feliform family Eupleridae, not included in the Herpestidae and Viverridae (Yoder et al., 2003). Therefore, the inversion detected in HJA, HPA and FFO could be a common cytogenetic signature for the Herpestidae and Eupleridae.

In Viverridae, only one inversion (CFA 11/3/2/11/4/35) in VIN chromosome 6 (equivalent to FCA Alq, Figure 6a) was detected by CFA painting probes.

In Hyaenidae, three inversions (CFA 27/30/23/35, CFA 21/5/21/5/18 and CFA 5/9/5) were found in chromosomes homologous to FCA C2, D1, E1 in CCR $(2 n=40)$ (Perelman et al., 2005).

In Ursidae, the pattern of CFA paints 1, 2 and 5 on the homologues to FCA E2 was the same (CFA 1/5/2) in AME (Figure 4), TOR (Yang and Graphodatsky, 2004) and HMA (Tian et al., 2004), while the painting pattern of CFA paints 1, 2 and 5 on the equivalents of FCA E2 in other carnivores was CFA 1/2/5. Although chromosome equivalents of FCA E2 in TOR and HMA had a different centromere position, this inversion has been proposed as one common character for Ursidae (Tian et al., 2004). Another two inversions (CFA 35/4/11/2/3 and CFA $19 / 33 / 36 / 28$ ) were found in the genomes of AME (chromosome 3, equivalent to FCA Alq, Figure 4) and TOR (chromosome 6, equivalent to FCA C1q, Yang and Graphodatsky, 2004). 
Table 1 Inversions revealed by dog paint probes in large chromosome segments of conserved synteny in different carnivores

\begin{tabular}{|c|c|c|c|c|c|c|c|c|c|c|c|c|c|}
\hline & \multirow[t]{2}{*}{ Species (2n) } & \multicolumn{11}{|c|}{ Homologous cat chromosomes or chromosome segments } & \multirow[t]{2}{*}{ References } \\
\hline & & $A 1 q$ & $A 2 q$ & $B 1$ & B2 & $B 4$ & $c 1 q$ & $C 2$ & $D 1$ & D3 & $E 1$ & $E 2$ & \\
\hline \multirow{8}{*}{ Feliformia } & FCA (38) & & & 0 & & & & & & & & & Yang et al. (2000) \\
\hline & PLE (38) & & & 0 & & & & & & & & & Tian et al. (2004) \\
\hline & NNE (38) & & & 0 & & & & & & & & & Tian et al. (2004) \\
\hline & HJA (36) & & $\bullet$ & & & & & & & & & & This study \\
\hline & Viverridae & & & & & & & & & & & & \\
\hline & VIN (36) & $\mathbf{\square}$ & & & & & & & & & & & This study \\
\hline & Hyaenidae & & & & & & & & & & & & \\
\hline & CCR (40) & & & & & & & $\nabla$ & $\star$ & & $\llbracket$ & & Perelman et al. (2005) \\
\hline \multirow[t]{8}{*}{ Caniformia } & Ursidae & & & & & & & & & & & & \\
\hline & Ailuridae & & & & & & & & & & & & \\
\hline & $\mathrm{AFU}^{*}(36)$ & & & $\int$ & & $\oint$ & $\#$ & $※$ & & & & & Tian et al. (2002) \\
\hline & Procyonidae & & & & & & & & & & & & \\
\hline & PLO (38) & & & & & & & & & 【 & & & This study \\
\hline & Mustelidae & & & & & & & & & & & & \\
\hline & MVI (30) & $\square$ & & ๑ & $\S$ & $\triangle$ & $\star$ & $\boldsymbol{\nabla}$ & & & & & $\begin{array}{l}\text { Graphodatsky et al. } \\
\text { (2000b) }\end{array}$ \\
\hline & MFO (38) & घ & & $\odot$ & $\S$ & $\Delta$ & $\star$ & $\boldsymbol{\nabla}$ & & & & & This study \\
\hline
\end{tabular}

Abbreviations: AFU, Ailurus fulgens; AME, Ailuropoda melanoleuca; CCR, Crocuta crocuta; FCA, Felis catus; HJA, Herpestes javanicu; HMA, Helartos malayanus; LLY, Lynx lynx; MFO, Martes foina; MVI, Mustela vision; NNE, Neofelis nebulosa; PLE, Panthera leo; PLO, Procyon lotor; TOR, Tremarctos ornatus; VIN, Viverricula indica.

AFU*, inversions each was detected to occur between two chromosome segments (homologous to FCAA1p+C1q and FCAA2p+C2) in red panda. Different symbols indicated different inversions.

Identical inversion in different species was indicated by the same symbol. $\mathbf{0}$, CFA 11/3/2/11/4/35; $\diamond$, CFA 35/4/11/2/3; $\square$, CFA 3/2/11/4/35; $\bullet$, CFA 14/18/14/16; O, CFA 16/28/16/28/15/19/

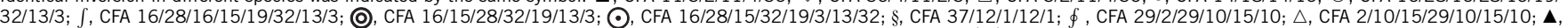

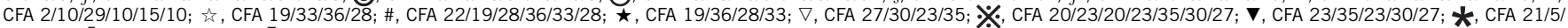
21/5/18; 【, CFA 26/7/26/7/1; 【, CFA 5/9/5; $\bullet$, CFA 1/5/2.

In Ailuridae, four inversions (CFA 20/23/20/23/35/30/27, CFA 22/ $19 / 28 / 19 / 36 / 33 / 28$, CFA 16/28/16/15/19/32/13/3 and CFA 29/2/29/10/ $15 / 10$ ) were detected in chromosomes homologous to FCA A2p+C2, $\mathrm{A} 1 \mathrm{p}+\mathrm{C} 1 \mathrm{q}, \mathrm{B} 1$ and $\mathrm{B} 4$ in $\mathrm{AFU}(2 n=36)$ by CFA paints (Tian et al., 2002).

In Procyonidae, the painting pattern generated by CFA chromosomes 1, 7 and 26 paints on PLO chromosome 12 (equivalent to FCA D3) was CFA 26/7/26/7/1 (Figure 2b), which was different from the pattern CFA $26 / 7 / 1$ on the equivalent chromosomes of other carnivores, suggesting that an inversion occurred in PLO chromosome 12.

In Mustelidae, inversions were detected in six homologous chromosomes or chromosomal segments (equivalents to FCA A1q, B1, B2, B4, C1q and C2) in MFO $(2 n=38$, Figure 2a) and MVI $(2 n=30$, Graphodatsky et al., 2000b) by CFA painting probes. Three common inversions (CFA 37/12/1/12/1, CFA 19/36/28/33 and CFA 23/35/23/30/ 27) were recognized in chromosomes of MFO and MVI homologous to FCA B2, C1q and C2. These inversions could be common cytogenetic signatures for the Mustelidae. In addition, the probe from CFA chromosome 10 gave three signals on chromosomes homologous to FCA B4 in MFO (Figure 1c) and MVI (Graphodatsky et al., 2000b). This inversion seems to be a common character for MFO and MVI. But another inversion revealed by the probe of CFA chromosome 15 was also found on the same chromosome in MVI, resulting in the different hybridization patterns on the homologues to FCA B4 in MFO and MVI.
After comparing inversions occurring in species from different families, we found an obviously identical inversion in a Feliformia (VIN) and a Caniformia species (MFO) (Table 1). A probe from CFA chromosome 11 painted two segments on VIN 6 and MFO 5 (equivalents to FCA Alq), resulting in the change of the CFA probes hybridization pattern on chromosomes homologous to FCA Alq from CFA $11 / 2 / 3 / 4 / 35$ in other carnivores to CFA $11 / 3 / 2 / 11 / 4 / 35$ in VIN and MFO, and MFO 5 and VIN 6 showed similar G-bands (Figures 2a and 6a). Nevertheless, it is difficult to say if this inversion is a common character for Feliformia and Caniformia as it could have evolved independently in these two different clades.

To sum up, inversions have been revealed in most carnivores studied by CFA paints, and some could represent cytogenetic signatures for a given carnivore species group, while others were specific for a given species. For instance, one inversion on the homologues to FCA A2q appears to be common to all species so far studied in the Herpestidae and Eupleridae; one inversion on the homologues to FCA B1 seems to be specific for all studied Felidae species, one inversion on the homologues to FCA E2 seems to be common to all species studied in Ursidae, and three common inversions on the homologues to FCA B2, C1q and C2 appear to be shared by two Mustelidae species. Our findings suggest that inversions have had an important role in the karyotype divergence of carnivores and, in particular, in species with synteny-conserved karyotypes such as the mustelids. 


\section{Mapping chromosome rearrangements onto the phylogenetic tree} of Carnivora

On the basis of the multiple nuclear gene sequences, Eizirik et al. (2010) proposed a complete molecular phylogeny for 50 different genera representing all carnivoran families and constructed a molecular timescale for the evolution of Carnivora. Many species with painting data were also included in this study. Mapping the chromosome rearrangements identified by chromosomal painting onto the relevant lineages of the phylogenetic tree proposed by Eizirik et al. (2010) enabled us to trace the characteristic chromosomal rearrangements and karyotypic evolution relationships in the major phylogenetic lineages in the order Carnivora (Figure 7).

Here, we used the $2 n=42$ ACK as the starting point to map the chromosome rearrangements that have occurred during the divergence of Carnivora. In Feliformia, except for the African palm civet (Nandinia binotata, NBI, Nandiniidae), the fission of ACK 1 (FCA $\mathrm{A} 2 \mathrm{p}+\mathrm{C} 2)$ was the common character for all the species studied. Two fusions (ACK8+15 and 10+18) differentiated the karyotype of NBI from that of other species in Feliformia. Three fusions (ACK $1 \mathrm{p}+9$, $3+15$ and $8+10$ ) and one inversion (ACK2) characterized the Felidae branch. One fission (ACK 7) supported the clade of Hyaenidae+Herpestidae+Eupleridae. A further inversion (ACK9) linked Herpestidae and Eupleridae. In Viverridae, different chromosome rearrangements were found in species with different diploid numbers.

In Caniformia, $>40$ fissions differentiated the karyotypes of species in Canidae from the ACK. Sixteen fissions and one inversion were common to all species studied in Ursidae. A further 16 fusions and 1 inversion occurred in AME. Two fusions and two fissions were common to TOR and bears with $2 n=74$. A further 11 fusions and 1 inversion differentiated the karyotype of TOR from that of other bears. Two common fusions (ACK8+15 and 10+18) were found in species in Mustelidae, Procyonidae, Ailuridae and Phocidae. They could be considered as common characters for species in the Arctoidea excluding Ursidae. Mephitidae (skunks) are the third family of Carnivora that was found to have highly rearranged karyotypes, besides Canidae and Ursidae (Perelman et al., 2008). More than 10 rearrangements differentiated the karyotypes of species in Mephitidae from the ACK. Species in Procyonidae have the same diploid number and similar painting patterns with some species in Mustelidae, supporting the idea that these two families have close relationships. The karyotypic evolutionary relationships among species in Mustelidae have been detailed by Graphodatsky et al. (2002). Furthermore, three common inversions were revealed in two species from the Mustelidae in our study. Some specific chromosomal rearrangements were found in species from the Phocidae and Ailuridae (Frönicke et al., 1997; Nie et al., 2002; Tian et al., 2002). The role of chromosome rearrangements in speciation remains controversial, but some landmark chromosomal rearrangements have been found at the major nodes of the Carnivora phylogenetic tree.

\section{DATA ARCHIVING}

This article does not report new empirical data or software.

\section{CONFLICT OF INTEREST}

The authors declare no conflict of interest.

\section{ACKNOWLEDGEMENTS}

We are grateful to PCM O'Brien (Cambridge Resource Centre for Comparative Genomics, Department of Veterinary Medicine, University of Cambridge) for her help in polishing the English during the preparation of the paper. This work was partly supported by grants from the National Natural Science Fund for
Distinguished Young Scholars in China and the Wellcome Trust (WT077008) to FY, the Ministry of Science and Technology of China to WN (No. 2005DKA21502), the State Key Laboratory of Genetic Resources and Evolution to DW (GREKF08-06), and MCB and SB RAS Integration Program to ASG and PP FY currently is an employee of the Wellcome Trust Sanger Institute.

Arnason U (2006). Order cetartiodactyla, cetacea. In: O'Brien SJ, Menninger JC, Nash WG (eds). Atlas of Mammalian Chromosomes. John Wiley and Sons Publishers: New York, pp 541-563.

Bielec PE, Gallagher DS, Womack JE, Busbee DL (1998). Homologies between human and dolphin chromosomes detected by heterologous chromosome painting. Cytogenet Cell Genet 81: 18-26.

Biltueva LS, Yang F, Vorobieva NV, Graphodatsky AS (2004). Comparative map between the domestic pig and dog. Mamm Genome 15: 809-818.

Breen M, Bullerdiek J, Langford CF (1999a). The DAPI banded karyotype of the domestic dog (Canis familiaris) generated using chromosome-specific paint probes. Chromosome Res 7: 401-406.

Breen M, Thomas R, Binns MM, Carter NP, Langford CF (1999b). Reciprocal chromosome painting reveals detailed regions of conserved synteny between the karyotypes of the domestic dog (Canis familiaris) and human. Genomics 61: 145-155.

Cavagna P, Menotti A, Stanyon R (2000). Genomic homology of the domestic ferret with cats and humans. Mamm Genome 11: 866-870.

Dutrillaux B, Couturier J (1983). The ancestral karyotype of Carnivora: comparison with that of platyrrhinus monkeys. Cytogenet Cell Genet 35: 200-208.

Eizirik E, Murphy WJ, Koepfli K-P, Johnson WE, Dragoo JW, Wayne RK et al. (2010). Pattern and timing of diversification of the mammalian order Carnivora inferred from multiple nuclear gene sequences. Mol Phylogenet Evol 56: 49-63.

Frönicke L, Müller-Navia J, Romanakis K, Scherthan H (1997). Chromosomal homeologies between human, harbor seal (Phoca vitulina) and the putative ancestral carnivore karyotype revealed by Zoo-FISH. Chromosoma 106: 108-113.

Graphodatsky AS, Perelman PL, Sokolovskaya NV, Beklemisheva VR, Serdukova NA, Dobigny $\mathrm{G}$ et al. (2008). Phylogenomics of the dog and fox family (Canidae, Carnivora) revealed by chromosome painting. Chromosome Res 16: 129-143.

Graphodatsky AS, Yang F, O'Brien PC, Perelman P, Milne BS, Serdukova N et al. (2001). Phylogenetic implications of the 38 putative ancestral chromosome segments for four canid species. Cytogenet Cell Genet 92: 243-247.

Graphodatsky AS, Yang F, O'Brien PC, Serdukova N, Milne BS, Trifonov V et al. (2000a). A comparative chromosome map of the Arctic fox, red fox and dog defined by chromosome painting and high resolution G-banding. Chromosome Res 8: 253-263.

Graphodatsky AS, Yang F, Perelman P, O'Brien PCM, Serdukova N, Milne BS et al. (2002). Comparative molecular cytogenetic studies in the order Carnivora: mapping chromosomal rearrangements onto the phylogenetic tree. Cytogenet Genome Res 96: 137-145.

Graphodatsky AS, Yang F, Serdukova N, Perelman P, Zhdanova NS, Ferguson-Smith MA (2000b). Dog chromosome-specific paints reveal evolutionary inter- and intrachromosomal rearrangements in the American mink and human. Cytogenet Cell Genet 90: 275-278.

Hameister H, Klett C, Bruch J, Dixkens C, Vogel W, Christensen K (1997). Zoo-FISH analysis: the American mink (Mustela vison) closely resembles the cat karyotype. Chromosome Res 5: 5-11.

Kulemzina Al, Trifonov VA, Perelman PL, Rubtsova NV, Volobuev V, Ferguson-Smith MA et al. (2009). Cross-species chromosome painting in Cetartiodactyla: Reconstructing the karyotype evolution in key phylogenetic lineages. Chromosome Res 17: 419-436.

Murphy WJ, Eizirik E, Johnson WE, Zhang YP, Ryder OA, O'Brien SJ (2001b). Molecular phylogenetics and the origins of placental mammals. Nature 409: 614-618.

Murphy WJ, Stanyon R, O'Brien SJ (2001a). Evolution of mammalian genome organization inferred from comparative gene mapping. Genome Biol 2: REVIEWS 0005.

Nash WG, Menninger JC, Padilla-Nash HM, Stone G, Perelman PL, O'Brien SJ (2008). The ancestral carnivore karyotype $(2 n=38)$ lives today in ringtails. J Hered 99: 241-253.

Nash WG, Menninger JC, Wienberg J, Padilla-Nash HM, O'Brien SJ (2001). The pattern of phylogenomic evolution of the Canidae. Cytogenet Cell Genet 95: 210-224.

Nash WG, O'Brien SJ (1987). A comparative chromosome banding analysis of the Ursidae and their relationship to other carnivores. Cytogenet Cell Genet 45: 206-212.

Nash WG, Wienberg J, Ferguson-Smith MA, Menninger JC, O'Brien SJ (1998). Comparative genomics: tracking chromosome evolution in the family Ursidae using reciprocal chromosome painting. Cytogenet Cell Genet 83: 182-192.

Nie W, Wang J, O'Brien PC, Fu B, Ying T, Ferguson-Smith MA et al. (2002). The genome phylogeny of domestic cat, red panda and five mustelid species revealed by comparative chromosome painting and G-banding. Chromosome Res 10: 209-222.

Nie W, Wang J, Perelman P, Graphodatsky AS, Yang F (2003). Comparative chromosome painting defines the karyotypic relationships among the domestic dog, Chinese raccoon dog and Japanese raccoon dog. Chromosome Res 11: 735-740.

Perelman PL, Graphodatsky AS, Dragoo JW, Serdyukova NA, Stone G, Cavagna P et al. (2008). Chromosome painting shows that skunks (Mephitidae, Carnivora) have highly rearranged karyotypes. Chromosome Res 16: 1215-1231.

Perelman PL, Graphodatsky AS, Serdukova NA, Nie W, Alkalaeva EZ, Fu B et al. (2005). Karyotypic conservatism in the suborder Feliformia (Order Carnivora). Cytogenet Genome Res 108: 348-354. 
Rettenberger G, Klett C, Zechner U (1995). Zoo-FISH analysis: cat and human karyotypes closely resemble the putative ancestral mammalian karyotype. Chromosome Res 3: 479-486.

Sargan DR, Yang F, Squire M, Milne SB, O’Brien PCM, Ferguson-Smith MA (2000). Use of flow-sorted canine chromosomes in the assignment of canine linkage, radiation hybrid and syntenic groups to chromosomes: refinement and verification of the comparative chromosome map for dogs and humans. Genomics 69: 182-195.

Stanyon R, Bigoni F, Wienberg J, Hadidian J (1993). A standardized G-banded karyotype for the raccoon (Procyon lotor) compared with the domestic cat. Boll Zool 60: 41-45.

Telenius H, Pelmear AH, Tunnacliffe A, Carter NP, Behmel A, Ferguson-Smith MA et al. (1992). Cytogenetic analysis by chromosome painting using DOP-PCR amplified flowsorted chromosomes. Genes Chromosomes Cancer 4: 257-263.

Tian Y, Nie W, Wang J, Ferguson-Smith MA, Yang F (2004). Chromosome evolution in bears: reconstructing phylogenetic relationships by crossspecies chromosome painting. Chromosome Res 12: 55-63.

Tian Y, Nie WH, Wang JH, Yang YF, Yang FT (2002). Comparative chromosome painting shows the red panda (Ailurus fulgens) has a highly conserved karyotype. Yi Chuan Xue Bao 29: 124-127.

Wienberg J, Stanyon R, Nash WG, O'Brien PC, Yang F, O'Brien SJ et al. (1997). Conservation of human vs. feline genome organization revealed by reciprocal chromosome painting. Cytogenet Cell Genet 77: 211-217.

Wurster-Hill DH (1973). Chromosomes of eight species from five families of Carnivora. J Mammal 54: 753-760.

Wurster-Hill DH, Centerwall WR (1982). The interrelationships of chromosome banding patterns in canids, mustelids, hyena, and felids. Cytogenet Cell Genet 34: 178-192.

Wurster-Hill DH, Gray CW (1975). The interrelationships of chromosome banding patterns in procyonids, viverrids, and felids. Cytogenet Cell Genet 15: 306-331.

Yang F, Alkalaeva EZ, Perelman PL, Pardini AT, Harrison WR, O'Brien PCM et al. (2003). Reciprocal chromosome painting among human, aardvark, and elephant (superorder
Afrotheria) reveals the likely eutherian ancestral karyotype. Proc Natl Acad Sci USA 100: 1062-1066.

Yang F, Graphodatsky AS (2004). Integrated comparative genome maps and their implications for karyotype evolution of carnivores. In: Schmid M, Nanda I (eds). Chromosomes Today, Vol. 14, Kluwer Acad Publ: The Netherlands, pp 215-224.

Yang F, Graphodatsky AS, Li T, Fu B, Dobigny G, Wang J et al. (2006). Comparative genome maps of the pangolin, hedgehog, sloth, anteater and human revealed by cross-species chromosome painting: further insight into the ancestral karyotype and genome evolution of eutherian mammals. Chromosome Res 14: 283-296.

Yang F, Graphodatsky AS, O'Brien PCM, Colabella A, Solanky N, Squire M et al. (2000). Reciprocal chromosome painting illuminates the history of genome evolution of the domestic cat, dog and human. Chromosome Res 8: 393-404.

Yang F, O'Brien PC, Milne BS, Graphodatsky AS, Solanky N, Trifonov V et al. (1999). A complete comparative chromosome map for the dog, red fox, and human and its integration with canine genetic maps. Genomics 62: 189-202.

Yoder A, Burns MM, Zehr S, Delefosse T, Veron G, Goodman SM et al. (2003). Single origin of Malagasy Carnivora from an African ancestor. Nature 421: 734-737.

Yu L, Li QW, Ryder OA, Zhang YP (2004). Phylogenetic relationships within mammalian order Carnivora indicated by sequences of two nuclear DNA genes. Mol Phylogenet Evol 33: 694-705.

(c)

This work is licensed under the Creative Commons Attribution-NonCommercial-No Derivative Works 3.0 Unported License. To view a copy of this license, visit http:// creativecommons.org/licenses/by-nc-nd/3.0/ 